\title{
OPEN Improving heat and mass transfer rates through continuous drop-wise condensation
}

\begin{abstract}
Ali Alshehri ${ }^{1,2,3 凶}$, Jonathan P. Rothstein ${ }^{4} \&$ H. Pirouz Kavehpour ${ }^{1}$
Drop-wise condensation (DWC) has been the focus of scientific research in vapor condensation technologies since the 20th century. Improvement of condensation rate in DWC is limited by the maximum droplet a condensation surface could sustain and the frequency of droplet shedding. Furthermore, The presence of non-condensable gases (NCG) reduces the condensation rate significantly. Here, we present continuous drop-wise condensation to overcome the need of hydrophobic surfaces while yet maintaining micron-sized droplets. By shifting focus from surface treatment to the force required to sweep off a droplet, we were able to utilize stagnation pressure of jet impingement to tune the shed droplet size. The results show that droplet size being shed can be tuned effectively by tuning the jet parameters. our experimental observations showed that the effect of NCG is greatly alleviated by utilizing this technique. An improvement by multiple folds in mass transfer compactness factor compared to state-of-the-art dehumidification technology was possible.
\end{abstract}

Condensation is a perplexing problem to fully uncover, yet, its applications play crucial roles in industrial development ${ }^{1-5}$. In order to promote more efficient applications, improving the condensation process has been the focus of many scientific research. Various condensation heat transfer augmentation techniques have been utilized, these techniques can be classified to; passive $\mathrm{e}^{6-12}$; active $\mathrm{e}^{13-15}$; and compound techniques. Unlike passive, active techniques require external forces to be applied either on the heat transfer surface or working fluid. Unsurprisingly, passive techniques have caught major attention of the scientific community because of their easier implementation in existing industrial applications. Contrarily, active techniques require additional equipment to exert the required forces to improve the heat and mass transfer efficiencies. This, in turn, means higher capital expenditures and operating expenses. Observing the state-of-the-art literature, the most trending technology is altering surface wettability ${ }^{6}$. However, it is still under development due to the chemical and physical durability issues of coating techniques ${ }^{6,16-19}$.

Major research efforts have been focused on developing durable and cost effective coating techniques to promote drop-wise condensation (DWC) and mitigate the formation condensate films on surfaces, i.e. film-wise condensation (FWC). DWC has shown to possess at least an order of magnitude improved heat transfer coefficient compared to $\mathrm{FWC}^{20}$. This improvement is highly dependant on the shedding frequency and size of droplets on surfaces ${ }^{21}$. Droplets shedding has been achieved primarily by gravity assistance ${ }^{22-24}$, droplet jumping ${ }^{14,25-27}$, drag force ${ }^{28-32}$, or by capillary driven movement ${ }^{33,34}$. It has been widely accepted that droplets of diameters below 20 micron contribute about $80 \%$ of the total heat transfer to the surface ${ }^{35}$. This implies that removing droplets of higher diameters is preferred, otherwise, their higher thermal resistance and coverage area will impede further condensation. Combining superhydrophobic surfaces and a shedding mechanism might seem to be the obvious solution for achieving efficient DWC. However, superhydrophobic surfaces are characterized by their low nucleation site density for condensation and higher droplet thermal resistance, therefore presenting a conflicting purpose $^{19}$. Additionally, superhydrophobic surfaces have been shown to transition to a wetting state at higher surface subcooling degrees losing their advantage to shed smaller droplets ${ }^{36}$. Therefore, there is a high demand to sustain efficient DWC with innovative techniques that go beyond surface modifications.

An extremely important concern most passive augmentation techniques has not resolved is the degradation of heat/mass transfer coefficients caused by the existence of non-condensable gases (NCG) ${ }^{19,37,38}$. Experimentally, degassing prior to running condensers has been successful in alleviating the effect of $\mathrm{NCG}^{19,22}$. Despite the

\footnotetext{
${ }^{1}$ Mechanical and Aerospace Engineering Department, University of California, Los Angeles, CA 90095, USA. 'Mechanical Engineering Department, King Fahd University of Petroleum and Minerals, Dhahran 31261, Saudi Arabia. ${ }^{3}$ Interdisciplinary Research Center for Renewable Energy and Power Systems, KFUPM, Dhahran 31261, Saudi Arabia. ${ }^{4}$ Department of Mechanical and Industrial Engineering, University of Massachusetts Amherst, Amherst, Massachusetts 01003-2210, USA. ${ }^{\circledR}$ email: alshehri@kfupm.edu.sa
} 
A

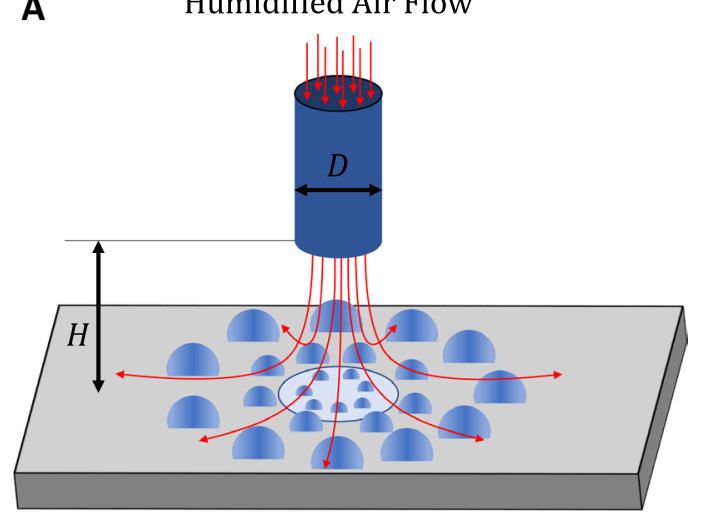

B

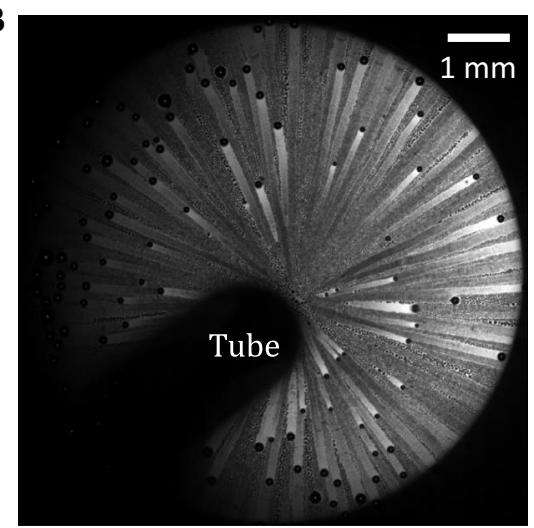

Figure 1. Utilizing Jet impingement as a means of continuous drop-wise condensation (CDC). (A) A schematic of CDC illustrating the condensation mechanism utilizing jet impingement as a means of enhanced condensation rate and droplet shedding. pure vapor or Humidified-air jet exits a tube of diameter $(D)$ at a standoff distance $(H)$ with a mean velocity $\left(v_{j}\right)$. (B) A still Microscopic image of CDC under a selected experimental condition $\left(D=0.047\right.$ inches, $H=0.32$ inches and $\left.R e_{j}=3600\left(v_{j}=45 \mathrm{~m} / \mathrm{s}\right)\right)$. Complete description of experimental set up is presented in methods section and supplementary material Fig. S1.

experimental convenience of such method, it is a highly impractical solution in large scale condensers. NCG can find their way into condensers via leak points or as chemical reaction products of vapor interacting with the equipment material ${ }^{39}$. On another front, the emerging humidification-dehumidification (HDH) desalination technology relies heavily on NCG as carrier gases. The premise of this technology is the low energy required to humidify air compared to other thermal desalination counterparts ${ }^{40-42}$. Even though it is evident that the dehumidifier in $\mathrm{HDH}$ technology is highly inefficient, the heat transfer deficit has been compensated by three alternatives. They are; (1) extended contact area ${ }^{43-46}$ (2) direct contact between humid air and cooling medium ${ }^{4-53}$; and (3) different NCG carrier ${ }^{54,55}$. Even though the former two solutions are promising, the latter seems to address the problem at its core, i.e. the effect of vapor diffusion coefficient thus condensation rate. Therefore, there is a pressing demand on working out a solution to enhance condensation with the presence of NCG.

To overcome the problem of sustaining efficient condensation without requiring unstable and expensive surface modifications, an active method needs to be designed. The active augmentation method needs to mitigate the negative effect of NCG while maintaining practical applicability. Several active methods have been tried, such as fluid/surface vibration ${ }^{56-59}$, electrohydrodynamic effects ${ }^{14,60-63}$, and rotating surfaces ${ }^{64-66}$, to name a few. The general goal of the different active methods is to prevent the condensate from growing by actively sweeping it off the surface. While this has shown to be effective, surface wettability is still important to generate DWC rather than FWC. In addition, the effect of NCG is still not resolved with the aforementioned methods. Here, we investigate utilizing jet impingement as an active method for providing DWC on surfaces with varying surface wettability. The jet impingement method not only helps with shedding droplets on wettable surfaces but also helps with mitigating the effect of NCG. The utilization of jet impingement in heat and mass transfer applications has been studied in heating/cooling for single phase flow ${ }^{67,68}$, drying application ${ }^{69}$, nucleate boiling ${ }^{70}$, and spray cooling ${ }^{71}$. Recently, on-demand impingement of pure steam jet has shown to alleviate the effect of NCG in accidental leakage ${ }^{72,73}$. Recently, it was shown that utilizing jet impingement in condensing water vapor in a humid environment results in a breath figure $\operatorname{spot}^{74}$. This spot defines the boundary over which effective condensation takes place.

To sustain efficient DWC without requiring unstable and expensive surface modifications, we present a novel 'continuous drop-wise condensation' (CDC) as a method to tune the maximum droplet size on modified and unmodified condensation surfaces. We also present CDC as a method to improve condensation with NCG by means of thinning the diffusion boundary layer and therefore reducing the resistance to diffusion. Impinging a modified or unmodified surface with a jet of humid air or pure vapor results not only in a higher heat and mass transfer coefficients but provides an excellent droplet shedding mechanism (Fig. 1). Controlling the diameter of droplet shedding is made possible by tuning the jet parameters, e.g. exiting diameter, velocity, and standoff distance. To provide evidence of the proposed mechanism, several experiments were conducted under various jet parameters as well as different surfaces with a wide range of advancing contact angles, i.e. $\theta_{A}=70^{\circ}-160^{\circ}$. In addition, we utilize our experimental observation to show that CDC provides over 8-fold improvement in compactness factor compared to state-of-the-art dehumidifiers. Furthermore, using an analytical model, we show that CDC provides enhancement in heat flux of over $300 \%$ compared to gravity-assisted shedding mechanisms. This is made possible by the improved mechanism of tuning the maximum droplet size compared to state-of-the-art techniques. Finally, We provide a theoretical framework for understanding droplet dynamics by comparing the different forces acting on a droplet during jet impingement. 


\section{Results}

Shedding of droplets under jet impingement. The dynamics of jet impingement on a surface is characterized by a stagnation region that spans almost two nozzle diameters ${ }^{75,76}$. Beyond this radial location, a wall jet forms that behaves similar to a Blasius boundary layer. Here we show that the force within the stagnation region provides an excellent shedding capability. To study this, we visualize under a microscope the growth and onset of shedding of droplets under different jet Reynolds numbers $R e_{j}=4 Q / \pi \nu D$, where $Q$ is the jet flow rate, $v$ is the kinematic viscosity of humid air, and $D$ is the tube exit diameter (Fig. 2). To provide consistent comparisons, the surfaces were cleaned prior to each experimental run as outlined in the methods section. Additionally, the advancing and receding contact angles were measured before and after each experimental run with no significant changes due to mobile droplet shearing effects. Here, we utilized a hydrophobic Si wafer $\left(\theta_{A}=107^{\circ}\right.$ and $\theta_{R}=103^{\circ}$ ) as the ${ }^{* *}$ condensation surface (see methods section and Table 1 ). The jet flow rate was first set to the desired value of jet mean velocity, after which the surface temperature was brought down to the desired temperature $\left(T_{s}=15^{\circ} \mathrm{C}\right)$. A high speed camera (Photron, FASTCAM Nova) attached to an optical microscope (Nikon, AZ100) was utilized to obtain videos and images of the condensation process (Video 3 and Fig. 2).

Figure 2, we show the quasi-steady droplet distribution on the condensation surface under different jet Reynolds numbers. In these experiments, the relative humidity of ambient air was $60 \%$ and the jet was $95 \%$. The tested surface was a hydrophobic Si wafer with an advancing contact angle of $\theta_{A}=107^{\circ}$ and a receding contact angle of $\theta_{R}=103^{\circ}$. Figure 2 and the corresponding Video 3 found in the supplemental material, the droplets can be observed to go through three main growth stages with time: Stage I - slow growth of stationary drops beneath the impinging jet; Stage II - fast growth as mobile droplets move radially outward merging with other droplets; and Stage III - slow growth of droplets that have come to rest far from the center of the impinging jet. In Stage I, single droplets residing on nucleation sites present on the surface initially start growing by direct condensation on their exposed surfaces. As single droplets reach a critical size $\left(R_{c} \sim 1 / \sqrt{4 N_{s}}\right.$, where $N_{s}$ is the nucleation site density), they start coalescing with neighboring droplets. The growth mechanism in Stage I can be described by a combination of direct condensation and coalescence with neighboring droplets. As droplets grow to another critical size, determined by the jet shedding capability, they start departing their equilibrium location on the surface. The onset of droplet departure is observed to be located near the stagnation region. This implies that droplets in this region possess higher growth rates and experience higher drag forces compared to droplet located further away. It is also observed that the critical droplet size at the onset of departure is reduced with increasing the jet Reynolds number. As droplets depart their first equilibrium location, they start moving radially outward coalescing with droplets in their path (Stage II). The action of movement and coalescence acts as an effective sweeping mechanism for improved DWC. The growth mechanism of a mobile droplet is determined only by coalescence and negligible direct condensation. After droplets move to locations further from the center of the impinging jet, the retention force due to surface tension overcomes the drag force by the gas flow around them and they decelerate and stop. Stationary far-field droplets can be observed clearly in Fig. 2A but are out of frame in Fig. 2B,C. Stationary droplets continue to grow by smaller mobile droplets that are generated from the stagnation region following their mother droplet path (stage III).

It is also worth noting that as droplets move along their radial path, they leave dry traces which appear as white traces under the microscope. The process of droplet growth then repeats on the traces until another droplet coming from the stagnation region sweeps it away. Under ideal circumstances, the size of the stationary droplets within these traces is limited by the size of the mobilized droplets. Hence, a mobilized droplet can be thought of as the maximum size a droplet can grow. In some circumstances, surface imperfections or dust particles can interfere with the mobile droplet sweeping action but could be mitigated by cleaning the surface thoroughly.

Figure 2A-E, a magnified view of the droplets formed beneath the impinging jets are shown to illustrate the effect that jet velocity and jet Reynolds number have on droplet mobility. At the smallest Reynolds number presented in Fig. $2 \mathrm{~A}, R e_{j}=1200\left(v_{j}=15 \mathrm{~m} / \mathrm{s}\right)$, droplets were not observed to shed even as they grew quite large. Figure $2 \mathrm{~B}$, at a $R e_{j}=2400\left(v_{j}=30 \mathrm{~m} / \mathrm{s}\right)$, droplets with radii greater than $R_{\max }=33 \mu \mathrm{m}$ were observed to shed and move radially outward coalescing with smaller drops and growing as they moved. With increasing Reynolds number, a further reduction in shedding drop size was observed. For the highest flow rate tested, $R e_{j}=6000$ $\left(v_{j}=75 \mathrm{~m} / \mathrm{s}\right)$, droplets with radii greater than $R_{\max }=13 \mu \mathrm{m}$ were observed to shed. These drop sizes are significantly smaller than the case of gravity-assisted droplet shedding or the shearing effect of boundary layer flows where only droplets with radii above 250 microns shed from a hydrophobic surface ${ }^{33}$. Our results clearly demonstrate that the maximum condensed droplet size can be efficiently tuned by controlling the impinging jet velocity and Reynolds number.

Surface wettability is an important factor in determining the shedding capability of a surface regardless of the active mechanism generating the shedding forces. In order to test the effect of wettability on CDC, the results of a series of experiments are presented in Fig. 3 for five different surfaces with a wide range contact angles at a fixed jet Reynolds number of $R e_{j}=3600\left(v_{j}=45 \mathrm{~m} / \mathrm{s}\right)$. The details of the topography and chemistry of each surface are presented in the Materials and Methods section, and SEM images are shown in Fig. S2 (supplementary material).

In these experiments, ambient temperature was $21^{\circ} \mathrm{C}$ and the surface temperature $15^{\circ} \mathrm{C}$ while the relative humidity of ambient air and the jet were $60 \%$ and $95 \%$, respectively. The advancing, $\theta_{A}$, and receding contact angle, $\theta_{R}$, for each surface are presented in Table 1 along with the contact angle hysteresis, $\theta_{A}-\theta_{R}$. The condensation process and shedding capability is visualized in Fig. 3 with videos available as supplementary material (Videos 1-5). Two hydrophilic surfaces, one smooth and one microstructured, with different contact angles are presented in Fig. 3A and 3B. On the smooth hydrophilic surface, Fig. 3A, droplets with radii greater than $20 \mu \mathrm{m}$ were observed to shed. The microstructured hydrophilic surfaces has roughly twice the contact angle hysteresis of the smooth hydrophilic surface, $\theta_{A}-\theta_{R}=20^{\circ} \mathrm{vs} 13^{\circ}$. As a result, the mobility of the droplets is hindered by the increased interfacial pinning force on the droplets caused by the presence of the microstructures and an increase 


$$
\begin{gathered}
A R e_{j}=1200 \\
\left(v_{j}=15 \mathrm{~m} / \mathrm{s}\right)
\end{gathered}
$$
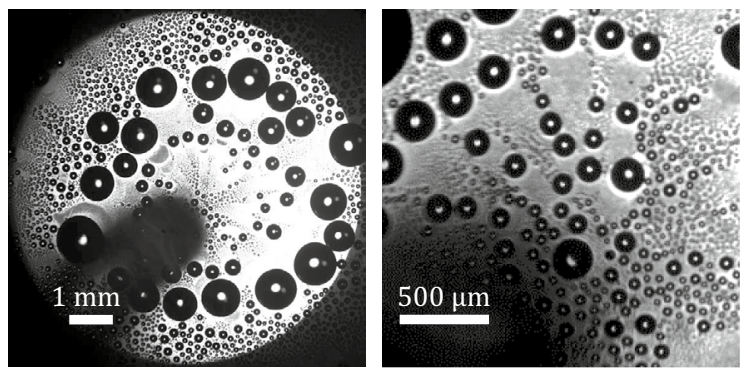

$$
\begin{gathered}
\text { B } R e_{j}=2400 \\
\left(v_{j}=30 \mathrm{~m} / \mathrm{s}\right)
\end{gathered}
$$
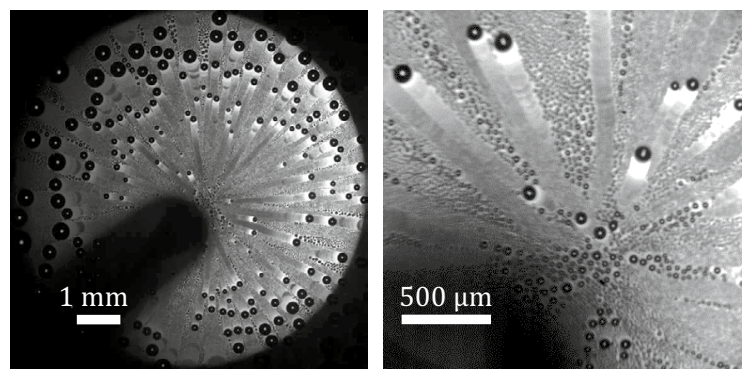

$$
\begin{gathered}
\text { C } R e_{j}=3600 \\
\left(v_{j}=45 \mathrm{~m} / \mathrm{s}\right)
\end{gathered}
$$
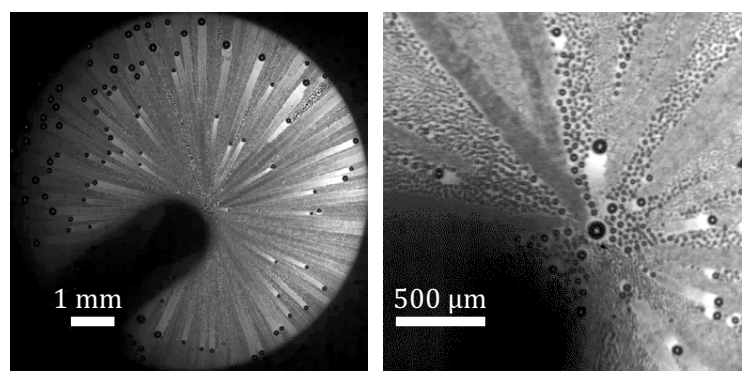

$$
\begin{gathered}
\text { D } R e_{j}=4500 \\
\left(v_{j}=60 \mathrm{~m} / \mathrm{s}\right)
\end{gathered}
$$
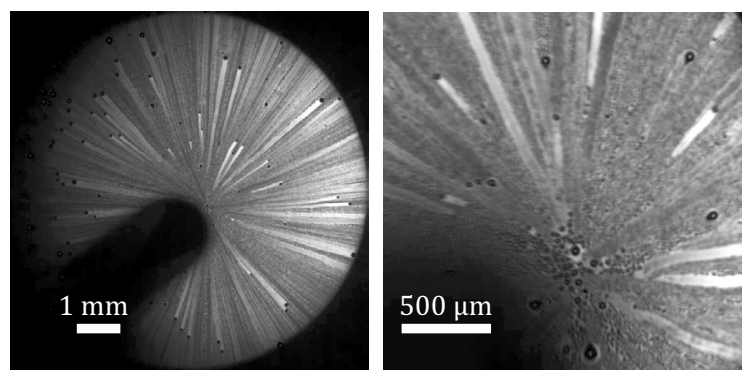

$$
\begin{gathered}
\mathrm{E} R e_{j}=6000 \\
\left(v_{j}=75 \mathrm{~m} / \mathrm{s}\right)
\end{gathered}
$$
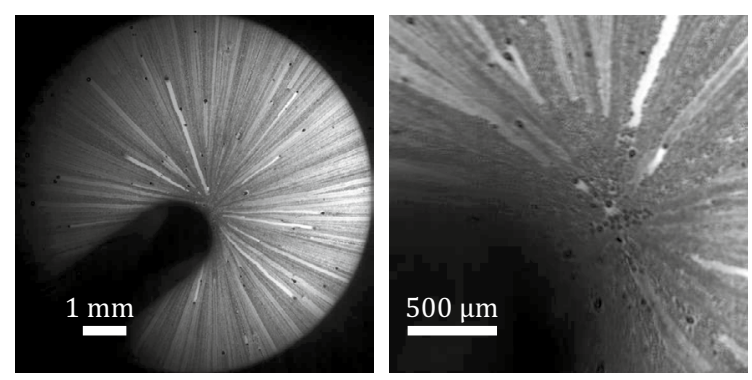

Figure 2. Effect of jet Reynolds number on the size of shed droplets. Images show results of condensation experiments performed at an ambient temperature of $21^{\circ} \mathrm{C}$ and surface temperature of $15^{\circ} \mathrm{C}$ where the relative humidity of ambient and jet were $60 \%$ and $95 \%$, respectively. The tested surface was a hydrophobic Si wafer with $\theta_{A}=107^{\circ}$ and $\theta_{R}=103^{\circ}$. Two different close up view are shown for condensation with jet Reynolds numbers of $(\mathbf{A}) R e_{j}=1200\left(v_{j}=15 \mathrm{~m} / \mathrm{s}\right),(\mathbf{B}) R e_{j}=2400\left(v_{j}=30 \mathrm{~m} / \mathrm{s}\right),(\mathbf{C}) R e_{j}=3600\left(v_{j}=45 \mathrm{~m} / \mathrm{s}\right),(\mathbf{D}) R e_{j}=4500$ $\left(v_{j}=60 \mathrm{~m} / \mathrm{s}\right)$, and $(\mathbf{E}) R e_{j}=6000\left(v_{j}=75 \mathrm{~m} / \mathrm{s}\right)$.

in the radius of the shedding drops was observed to a value of $36 \mu \mathrm{m}$. On the other hand, minimizing the contact angle hysteresis, as is done for both the hydrophobic and the nanostructured superhydrophobic surface shown in Fig. 3C,E, dramatically reduces the minimum droplet shedding radius by reducing the interfacial pinning force. 


\begin{tabular}{|c|c|c|c|c|}
\hline Name: description & $\theta_{A}$ & $\theta_{R}$ & $\theta_{A}-\theta_{R}$ & $\cos \left(\theta_{A}\right)-\cos \left(\theta_{R}\right)$ \\
\hline Surface 1: hydrophilic & $85 \pm 2^{\circ}$ & $72 \pm 2^{\circ}$ & $13 \pm 3^{o}$ & $0.22 \pm 0.05$ \\
\hline Surface 2: hydrophilic Microstructured & $70 \pm 2^{\circ}$ & $50 \pm 2^{o}$ & $20 \pm 3^{o}$ & $0.30 \pm 0.04$ \\
\hline Surface 3: hydrophobic & $107 \pm 2^{\circ}$ & $103 \pm 2^{\circ}$ & $4 \pm 3^{\circ}$ & $0.03 \pm 0.05$ \\
\hline Surface 4: superhydrophobic Microstructured & $160 \pm 2^{\circ}$ & $127 \pm 2^{\circ}$ & $33 \pm 3^{\circ}$ & $0.34 \pm 0.03$ \\
\hline Surface 5: superhydrophobic Nanostructured & $157 \pm 2^{\circ}$ & $154 \pm 2^{\circ}$ & $3 \pm 3^{o}$ & $0.022 \pm 0.02$ \\
\hline
\end{tabular}

Table 1. Advancing and Receding contact angles of the different surfaces used. Surfaces have different wettability and contact angle hysteresis.

A Surface 1:

Hydrophilic

$\left(\theta_{A} / \theta_{R}=85^{\circ} / 72^{\circ}\right)$

B Surface 2:

Hydrophilic Micro-

Structured

$\left(\theta_{A} / \theta_{R}=70^{\circ} / 50^{\circ}\right)$

\section{Surface 3: Hydrophobic \\ $\left(\theta_{A} / \theta_{R}=107^{\circ} / 103^{\circ}\right)$}

\section{Surface 4:}

Superhydrophobic MicroStructured

$$
\left(\theta_{A} / \theta_{R}=160^{\circ} / 127^{\circ}\right)
$$

\section{E Surface 5:}

Superhydrophobic

Nanostructured

$$
\left(\theta_{A} / \theta_{R}=157^{\circ} / 154^{\circ}\right)
$$
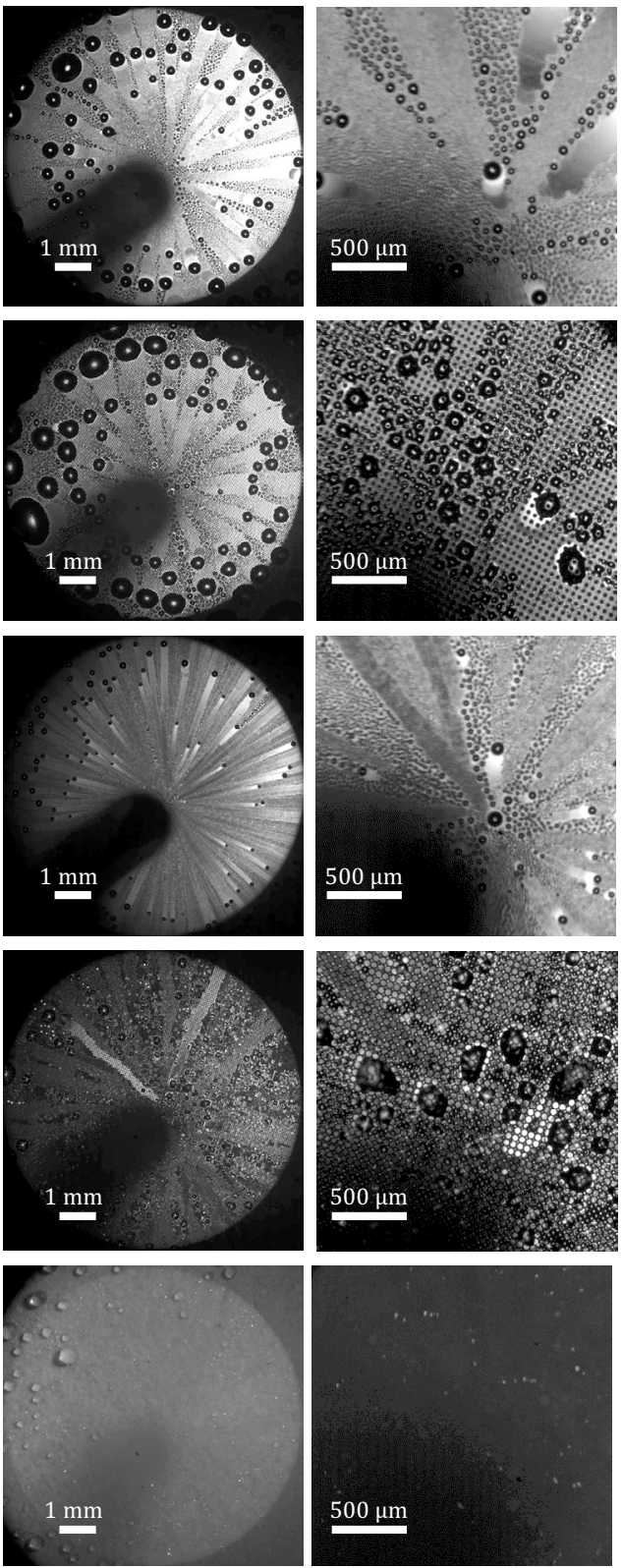

Figure 3. Effect of surface wettability on the condensation process. The condensation experiments were performed with an ambient air temperature of $21^{\circ} \mathrm{C}$ and surface temperature of $15^{\circ} \mathrm{C}$, while the relative humidity of ambient and jet were $60 \%$ and $95 \%$, respectively. Two different close up views are shown for condensation with varying surface wettability and contact angle hysteresis at a fixed jet Reynolds number of $R e_{j}$ $=3600\left(v_{j}=45 \mathrm{~m} / \mathrm{s}\right)$. The advancing and receding contact angles is displayed beside images of each surface. 
For example, droplet radius of the drops shedding from the hydrophobic surface in Fig. 3C was $13 \mu \mathrm{m}$. For the nanostructured superhydrophobic surface shown in Fig. 3E, the surface looks clear under the microscope with no evidence of the pathlines of shedding droplets clearly visible in Fig. 3A-C. This is probably due to the low condensation rate due in large part to the low density of nucleation sites on these nanostructured superhydrophobic surfaces, but it could also be the result of droplet jumping from the surface as they coalesce and interfacial energy is recovered in the form of kinetic energy. Some evidence for the presence of droplet jumping can be seen in the videos provided in the supplementary materials. Droplet jumping has been shown to improve the heat transfer to a surface during condensation ${ }^{25}$ and will be discussed in more detail later. Finally, we analyze the results of the microstructured superhydrophobic surface in Fig. 3D. Interestingly, even though it had the largest advancing contact angle, the microstructured superhydrophobic surface also had the highest contact angle hysteresis. The CDC experiments for this were characterized by a significant pinning of droplets and a very large variability and uncertainty in the size of the shedding droplets. As a result, the discussion of the drop dynamics that follows will be focused primarily on Surfaces 1,2 and 3 for which repeatable data could be obtained.

Dehumidification with CDC for improved heat and mass transfer rates. In order to experimentally evaluate the mass transfer coefficient, we utilized an optical method of observing the growth and departure of condensate droplets near the impingement region. The evolution of condensate volume with time was calculated within the impingement region (a surface with a diameter of $1 \mathrm{~mm}$, or $A=0.785 \mathrm{~mm}^{2}$ ). Additionally, the number and volume of droplets shedding and leaving the impingement region was tracked with time (Fig. S6). The mass transfer coefficient can be written as follows.

$$
h_{m}=\frac{\rho_{l}}{\left(\omega_{j}-\omega_{s}\right) A} \frac{d}{d t} \sum V_{d}
$$

where $\rho_{l}$ is the liquid density, $\omega_{j}$ is the vapor mass fraction at jet conditions, $\omega_{s}$ is the vapor mass fraction evaluated at the surface temperature and $d V_{d} / d t$ is the condensate volumetric growth per unit time. The volumetric growth rate can be calculated directly from the video images. To calculate the droplet volume, the droplets are assumed to take the form of a spherical cap because their radii are smaller than the capillary length. For a droplet that is a spherical cap with an optically observed radius of $R$ on the hydrophobic surface, the volume of a droplet can be calculated from

$$
V_{d}=\frac{\pi}{3}\left(2+\cos \theta_{A}\right)\left(1-\cos \theta_{A}\right)^{2} R^{3}
$$

The overall heat transfer coefficient is obtained by equating the heat transfer to the latent heat released during condensation as follows.

$$
U=\frac{\rho_{l} h_{f g}}{\left(\omega_{j}-\omega_{s}\right)\left(T_{j}-T_{s}\right) A} \frac{d V_{d}}{d t}
$$

where $h_{f g}$ is the latent heat of vaporization. Because droplets departing the impingement region collect more liquid as the travel radially outward, considering only the impingement region provides the lower limit of the condensation rates and the mass transfer coefficient (Fig. 4A). It should be noted that the growth rate of condensate mass was taken when a steady state condensation was reached, indicated by a linear growth with time (see Fig. S3). It is also important to mention that micro-droplets within the measurement surface that have not been mobilized by the jet drag force were not included in the calculation of the condensation rate until they are either mobilized or swept off by larger droplets.

Figure 4, results for the mass and heat transfer coefficients are presented for different jet Reynolds numbers. In these experiments, the relative humidity of ambient air was $60 \%$ and the jet was $95 \%$. The tested surface was a hydrophobic Si wafer with an advancing contact angle of $\theta_{A}=107^{\circ}$ and a receding contact angle of $\theta_{R}=103^{\circ}$. The mass transfer coefficient ranged from $0.2<h_{m}<3.6 \mathrm{~kg} / \mathrm{m}^{2} \mathrm{~s}$ while the heat transfer coefficient ranged from $0.4<U<7.2 \mathrm{~kW} / \mathrm{m}^{2} \mathrm{~K}$. The largest values of heat and mass transfer coefficient were found for the lowest Reynolds numbers tested with the data approaching constant value beyond $R e_{j}>2000$. The reason for the reduction in the mass transfer coefficient by increasing Reynolds number is probably a result of the reduced concentration of vapor reaching the cooled surface after exiting the tube ${ }^{74}$. In Fig. 4B, a comparison of the mass transfer coefficient for different dehumidifiers along with the current CDC method is presented for condensation on hydrophobic surfaces. Here a Reynolds number of $R e_{j}=3600\left(v_{j}=45 \mathrm{~m} / \mathrm{s}\right)$ was chosen to represent the data as it appear in the middle of the Reynolds number independent CDC operating window in Fig. 4A. Three stateof-the-art dehumidifiers are shown namely (1) bubble column dehumidifier ${ }^{51}$, (2) flat plate dehumidifier ${ }^{77},(3)$ moving liquid beads dehumidifier ${ }^{53}$. As seen in Fig. 4B, when compared against the selected case of $R e_{j}=3600$ $\left(v_{j}=45 \mathrm{~m} / \mathrm{s}\right)$, the mass transfer coefficient, $h_{m}$, obtained utilizing CDC exceeds the highest state-of-the-art dehumidifier by more than 6x. If we compare against the results for a Reynolds number of $R e_{j}=1200\left(v_{j}=15\right.$ $\mathrm{m} / \mathrm{s}$ ) CDC outperforms the state-of-the-art dehumidifier by $40 \times$. Thus, CDC provides an extremely large heat and mass transfer coefficients that with continued refinement we believe could provide a two-order of magnitude improvement of mass transfer coefficient compared to the current state-of-the-art dehumidifiers.

Importantly, heat and mass transfer coefficients alone may not be sufficient to evaluate the performance of different dehumidifiers because they do not take into account the size or footprint of the dehumidifier. In practice, dehumidifiers like bubble column dehumidifiers can have a very large mass transfer coefficient, but their large size can make implementation difficult. To understand the effect of size for different dehumidifier designs, we follow the work of Sadeghpour et al. ${ }^{53}$ and compare each dehumidifier design through their compactness factors. 

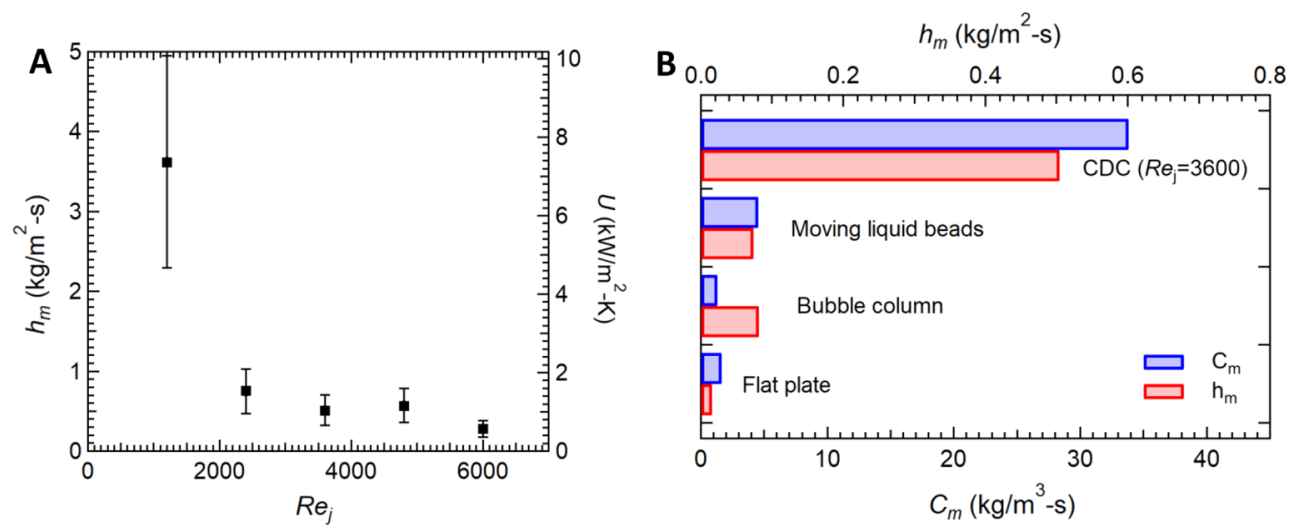

Figure 4. Heat and mass transfer rate improvement of CDC. (A) Experimental evaluation of mass transfer coefficient, $h_{m}$ of CDC as a function of impinging jet Reynolds number. The experiments were performed at an ambient temperature of $21^{\circ} \mathrm{C}$ and surface temperature of $15^{\circ} \mathrm{C}$ where the relative humidity of ambient and jet were $60 \%$ and $95 \%$, respectively. The tested surface was a hydrophobic Si wafer with $\theta_{A}=107^{\circ}$ and $\theta_{R}=$ $103^{\circ}$. The jet mean velocities were between $15 \mathrm{~m} / \mathrm{s}$ and $75 \mathrm{~m} / \mathrm{s}$. (B) Comparison of mass transfer coefficient and compactness factor for different state-of-the-art dehumidifiers; bubble column dehumidifier ${ }^{51}$, flat plate dehumidifier $^{77}$, moving liquid beads dehumidifier ${ }^{53}$.

The compactness factor indicates the heat or mass transfer rate per unit driving potential (temperature or vapor mass fraction) per unit volume and is defined as follows for mass transfer ${ }^{53}$

$$
C_{m}=\frac{h_{m} A}{V}
$$

Here, $C_{m}$ is the compactness factor of mass transfer unit, $A$ is the surface area over which measurement takes place, and $V$ is the volume of the dehumidification system. A schematic diagram of the CDC dehumidification system is presented in the supplemental materials in Fig. S1 so that the reader can better understand how the values for the volume of the CDC dehumidification system is evaluated to determine the compactness factor. Following the method used by Sadeghpour et al..$^{53}$, the the volume of the CDC dehumidification system is defined as

$$
V=A\left(H+H_{S i}+H_{A l}+H_{p p}\right)
$$

where $A$ is the surface area over which the condensation rate was evaluated, $H, H_{S i}, H_{A l}, H_{p p}$ are different heights in the system including the stand off distance of the nozzle, $H$, the thickness of the silicon wafer, $H_{S i}$, the thickness of the aluminum substrate, $H_{A l}$, and the thickness of the Peltier plate, $H_{p p}$, as depicted in Fig. S1. In table S1 of the supplementary material, we summarize the different parameters, including the area density, $A / V$, used here to calculated the compactness factor for each of the dehumidification techniques. It should be noted that, for the bubble humidifier, the transporting pipes were not considered part of the dehumidification system when calculating its volume $\mathrm{e}^{53}$.

The compactness factor for each of the dehumidification systems is presented alongside the mass transfer coefficient data in Fig. 4B. CDC is characterized by a large area density of $A / V=67 \mathrm{~m}^{-1}$. Only the flat plate dehumidifiers is larger with an area density of $108 \mathrm{~m}^{-1}$. Unfortunately, as seen in Fig. 4B, flat plate dehumidifiers have the lowest mass transfer coefficient by a significant margin. Thus, even with a large area density, the flat plate dehumidifier has a compactness factor that is only slightly better than the bubble column which is plagued by a small area density, $A / V=16 \mathrm{~m}^{-1}$, as described previously. As a result, when compared against the state-of-theart dehumidifiers, the CDC method described in this paper outperforms all other dehumidification techniques even when taking into consideration the relative size of the dehumidifier through the use of the compactness factor. In fact, when compared using the compactness factor, CDC outperforms the best state-of-the-art technique by a factor of more than $8 \mathrm{x}$. We expect that even higher values of compactness factor are possible through optimization of geometric parameters like nozzle standoff distance and impinging jet diameter and velocity.

Pure steam condensers with CDC for improved heat and mass transfer rates. We now turn to the case of utilizing our method to improve the heat and condensation rates for the case of pure vapor/steam. To characterize the condensation process due to the CDC improved droplet shedding, we visualized the condensation process on different jet Reynolds numbers (Fig. 2) and different surface wettability (Fig. 3). As observed, when droplets grow to their maximum droplet size near the stagnation region, they are shed by the jet impingement action. In contrast, in regular gravity-assisted DWC, the shedding mechanism is mainly due to the weight of the droplet. This requires droplets to grow by direct condensation and coalescence with neighboring droplets until reaching the capillary length ( $\mathrm{mm}$ range) beyond which their weight overcomes the surface tension force. For pure vapor condensation in a pure vapor environment, the high thermal resistance of large droplets reduces the heat transfer significantly (Fig.S3). In addition, allowing a surface to sustain high droplet size before shed- 

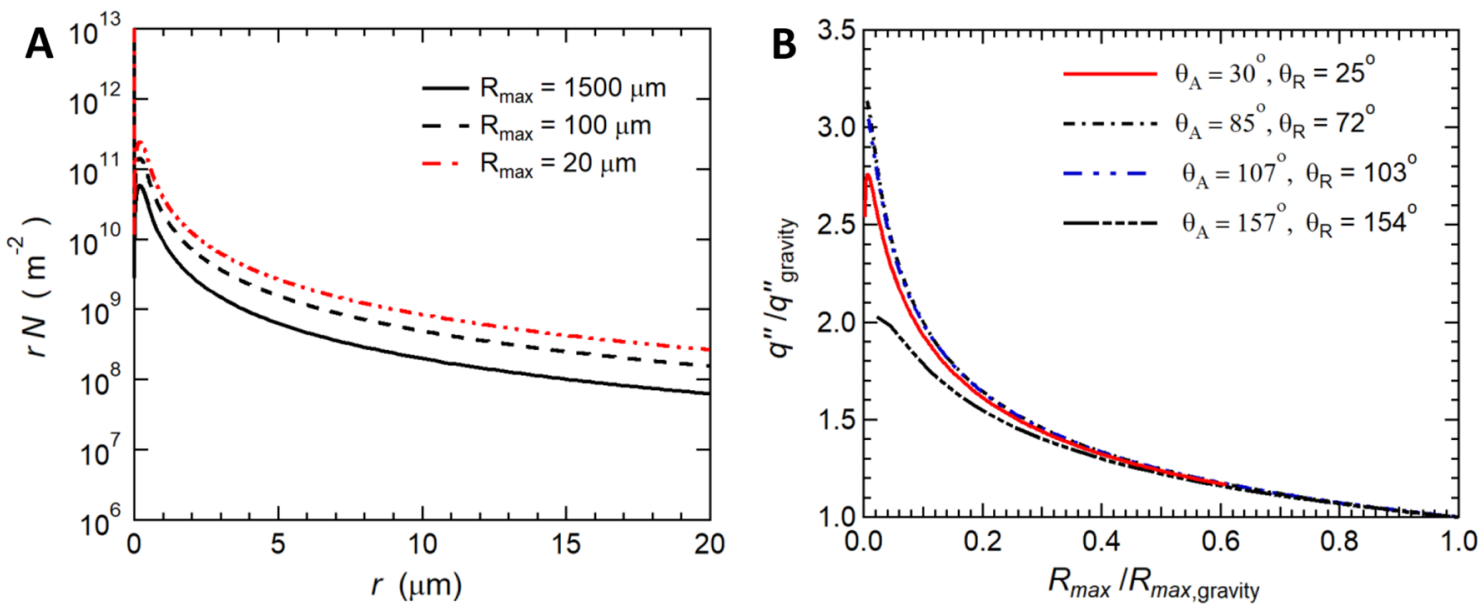

Figure 5. Heat transfer characterization of CDC. (A) The population density of droplets $(r N)$ of radii below $20 \mu \mathrm{m}$ for different maximum droplet radius $\left(R_{\max }\right)$ being shed by the jet impingement action. The ordinate is defined as the number of droplets per unit surface area. (B) Heat flux $\left(q^{\prime \prime}\right)$ to a surface exposed to pure vapor analytically evaluated at different maximum droplet radius $\left(R_{\max }\right)$. The heat flux and maximum droplet radius are normalized with values evaluated in case of gravity-assisted droplet shedding (see supplementary material section S-4).

ding results in a decrease in the small droplet density (Fig. 5A). It is well established that droplets with radii below $20 \mu \mathrm{m}$ contribute to almost $80 \%$ of the overall heat transfer to the surface ${ }^{35}$. Therefore, by reducing the maximum droplet size and increasing the population density of droplets below $20 \mu \mathrm{m} \mathrm{CDC}$ is an efficient means of increasing the heat and mass transfer rate to a surface.

It is possible to get a better understanding of the effect that the reduction in the maximum droplet size has on the heat flux using the analytical model developed by Rose et al. ${ }^{23,78}$. Although this model does not take into account the effects of shear flow in the vapor phase, we believe this model still provides a useful first order estimate of the effect of maximum drop diameter on heat flux. Unfortunately, an analytical model that incorporates all the physics of the CDC process is beyond the scope of this work. In the analytical model of Rose et al. model $^{23,78}$, the overall heat flux to a surface exposed to condensation in an environment of pure vapor is given as

$$
q^{\prime \prime}=\int_{r_{\min }}^{r_{e}} q_{d}(r, \theta) n(r, \theta) d r+\int_{r_{e}}^{r_{\max }} q_{d}(r, \theta) N(r, \theta) d r
$$

where $q_{d}(r, \theta)$ is the heat transfer through a single droplet with Radius $r$ and contact angle $\theta, n(r, \theta)$ and $N(r, \theta)$ are the population density of of small and large droplets, respectively. Droplet below the droplet interaction radius $\left(r_{e}=1 / \sqrt{4 N_{s}}\right)$ grow by direct condensation (small droplets) while droplet above the droplet interaction radius grow by direct condensation and coalescence with neighboring droplets (large droplets). The effect of maximum droplet radius can be seen in Fig. 5A. Figure 5A, it is shown that decreasing the maximum droplet radius can shift the droplet size distribution to smaller and smaller droplets. The total heat transfer from the surface can be calculated by integrating Eq. (5) given that the heat transfer through a single droplet is dependent on the drop size and can be written as

$$
q_{d}(r, \theta)=\pi r^{2}\left(T_{s a t}-T_{s}-\frac{2 T_{\text {sat }} \gamma}{\rho_{l} h_{f g} r}\right)\left(\frac{1}{2 h_{i}(1-\cos \theta)}+\frac{r \theta}{4 k_{l} \sin \theta}+\frac{\delta_{s}}{k_{s} \sin ^{2} \theta}\right)^{-1}
$$

For a detailed analysis of the analytical model, see the Supplementary material (section S-4).

As discussed above, the main difference between CDC and this analytical theory of Rose et al..$^{23,78}$ is the presence of a strong shear flow that dramatically reduces the maximum droplet radius, $r_{\text {max }}$, of the condensing water as as shown in Fig. 5B. The effect of maximum droplet radius is easily accounted for by changing the limit of integration in Eq. (5). Furthermore, because the jet advective transport does not introduce additional thermal resistance, the effect of the shear flow can be approximated as an enhanced gravitation body force that acts only to improve shedding. As a result modifications to Eq. (6) are not required to approximate the effect of maximum droplet radius on heat flux.

In Fig. 5B, the heat flux to a surface is plotted as a function of the maximum droplet radius radius for the four different surfaces studied here. In each case, the heat transfer rate is improved significantly by lowering the maximum droplet radius. For example, an improvement of the heat flux (or equivalently the condensation rate) of roughly $150 \%$ is predicted for a hydrophilic surface when the maximum droplet radius is reduced from that under gravity $\left(R_{\max }=1.5 \mathrm{~mm}\right)$ to that under CDC $\left(R_{\max }=20 \mu \mathrm{m}\right)$. If one compares different surfaces, an improvement of more than $375 \%$ can be achieved by changing from a hydrophilic surface to a superhydrophobic surface due to the decrease in maximum radius. This shows that heat transfer and consequently condensation rate can be improved by tuning the maximum droplet size utilizing CDC. 
A

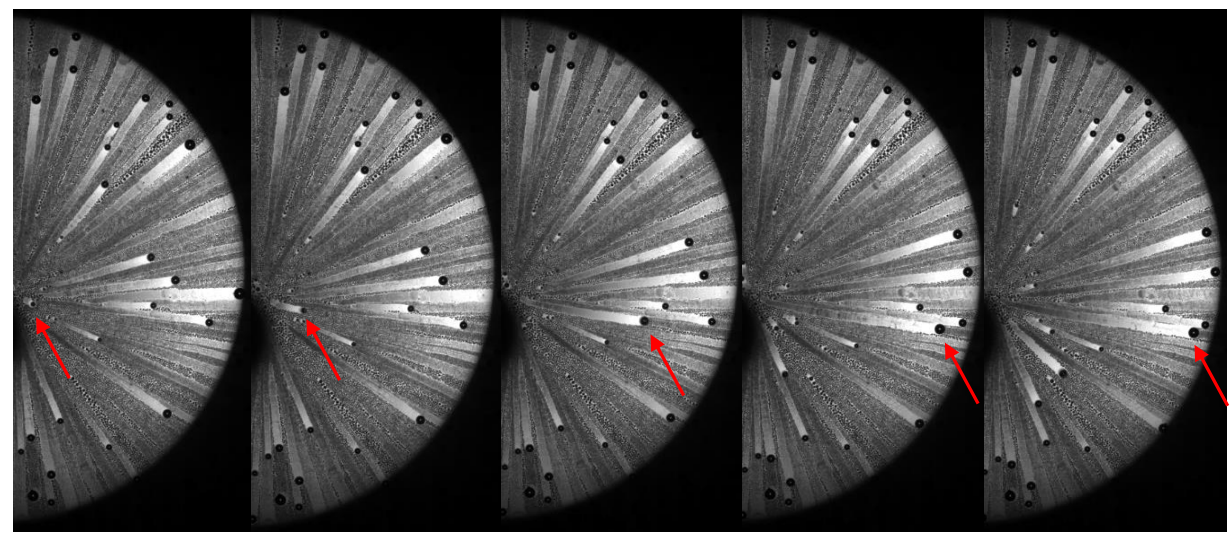

B

$$
t=80 \mathrm{~ms} \quad t=100 \mathrm{~ms} \quad t=120 \mathrm{~ms} \quad t=160 \mathrm{~ms} \quad t=180 \mathrm{~ms}
$$

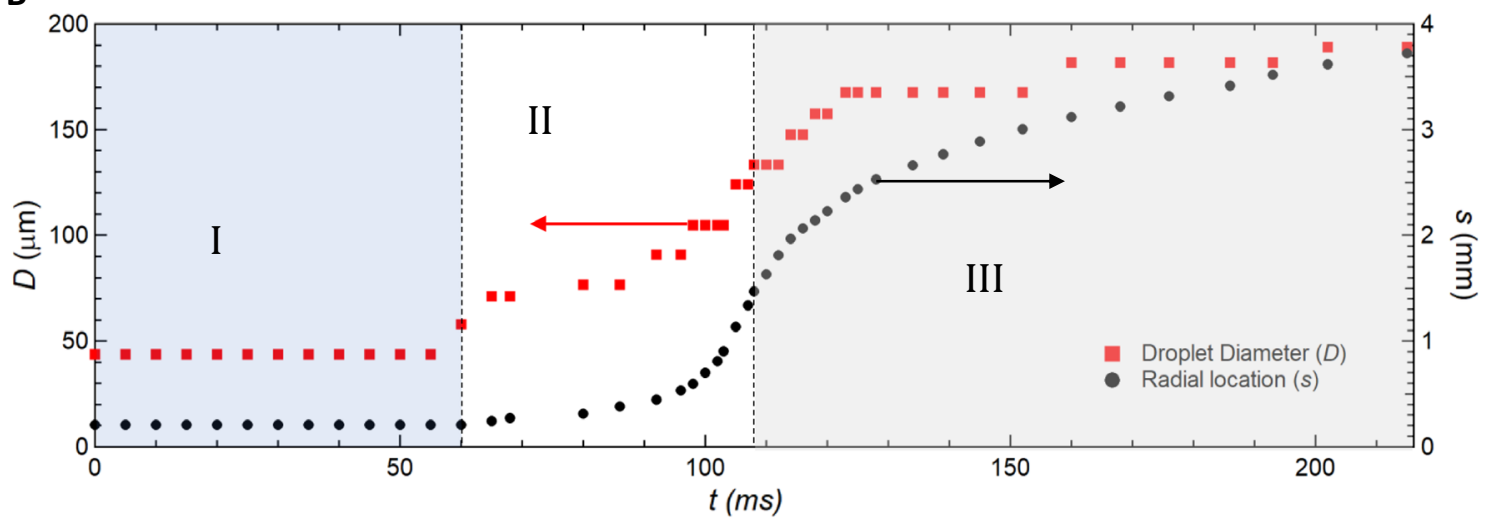

Figure 6. A typical time evolution of droplet motion and growth. (A) Time evolution of a droplet departing its first equilibrium location and moving radially outward. (B) Typical transience plot illustrating both the location of the droplet $(s)$ and its diameter $(D)$ as it coalesces with other droplets in its path. The droplet goes through three different periods; (I) a waiting period before droplet departure, (II) an accelerating droplet period, and (III) a decelerating droplet period. This plot is generated for a selected case of $R e_{j}=3600\left(v_{j}=45 \mathrm{~m} / \mathrm{s}\right)$ and hydrophobic surface $\left(\theta_{A}=107^{\circ}\right.$ and $\left.\theta_{R}=103^{\circ}\right)$.

Jet-droplet dynamics. The dynamics of droplet shedding and motion is governed by an interplay between droplet inertia, droplet retention forces due to surface tension, viscous friction within the drop, and drag force due to flow of air around the drop. The effect of each of these was investigated by tracking the motion and size of individual droplets at the different stages of growth and motion as they as they moved across different substrates under different impinging jet conditions. In Fig. 6A, a sample droplet is traced with time along the hydrophobic surface $\left(\theta_{A}=107^{\circ}\right.$ and $\left.\theta_{R}=103^{\circ}\right)$ for one representative case at a jet Reynolds number of $R e_{j}=3600\left(v_{j}=45\right.$ $\mathrm{m} / \mathrm{s})$. The diameter and location of the droplet is plotted as functions of time in Fig. 6B. Three different periods can be clearly distinguished from the data: Period I - a waiting period before droplet departure; Period II - a period of radial acceleration of the droplet; and Period III - a period of droplet deceleration until the droplet comes to rest. The onset of droplet motion occurs after a waiting period during which the droplet grows through condensation to a critical size. At this critical size, the aerodynamic drag force acting on the droplet becomes larger than the interfacial retention forces acting along the contact line between the droplet and the surface. After the droplet departs its initial location (onset of period II), it coalesces with droplets in its path resulting in a fast growth in droplet size and an acceleration across the surface because with increasing size the aerodynamic drag force grows faster with droplet radius than then the interfacial retention force $\left(R^{2}\right.$ vs. $\left.R\right)$. During period II, the droplet roughly triples meaning the volume has increased by a factor close to thirty. During period III, the droplet decelerates and the rate of diameter growth slows as fewer coalescence event occur. This deceleration occurs because the strength of the shear flow near the wall decreases as the drop moves radially outward from the center of the impinging jet $(1 / s)$. Qualitatively similar results were observed for all surfaces tested provided the jet Reynolds number was larger than the critical Reynolds number to initiate droplet motion.

Figure 7A, the droplet location $(s)$ is shown as a function of elapsed time $\left(t-t_{o}\right)$ for four different jet Reynolds numbers $\left(R e_{j}\right)$ for the hydrophobic surface, where $t_{o}$ is the time of onset of motion. The different curves show similar trends depicted in Fig. 6B and the existence of three different motion periods. Note that the transition from period II to period III is delayed by lowering the jet Reynolds number. Additionally, the droplet begins to decelerate at a location further from the center of the impinging jet for larger jet Reynolds numbers. Ideally, we would like to collapse all of these curves onto a single master curve. The obvious choice is non-dimesionalize the time on the x-axis with the jet velocity divided by the jet diameter, $\left(t-t_{0}\right) V_{j} / D_{j}$. Unfortunately, this simple non-dimensionalization does not collapse the data. Nor will the data collapse if the critical diameter for droplet 

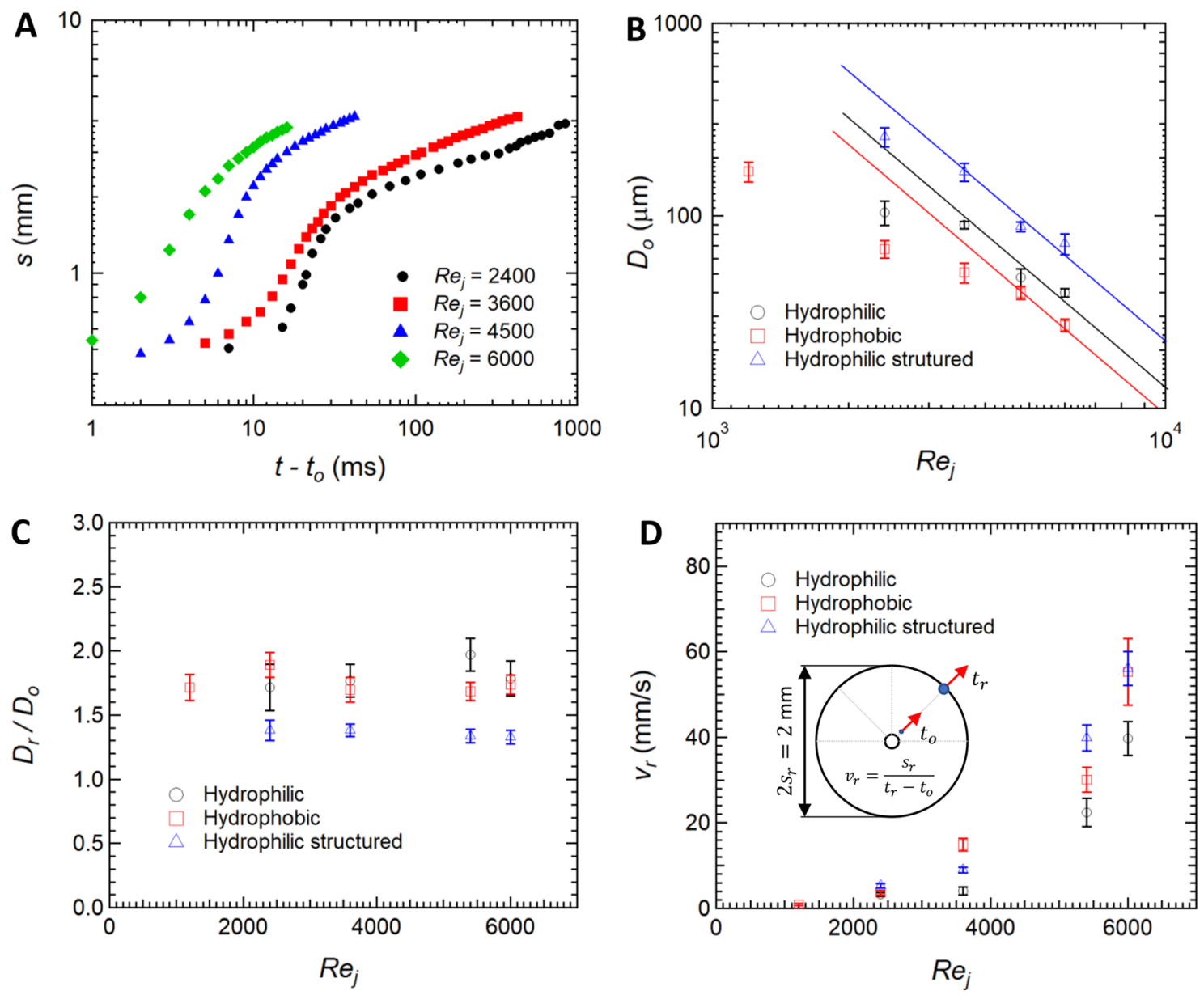

Figure 7. Dynamics of droplet-jet interaction. (A) Droplet location from the center of the impingement region $(s)$ as a function of time $\left(t-t_{o}\right)$, where $t_{o}$ is the time of onset of motion. The plot shows the effect of different jet Reynolds numbers $\left(R e_{j}\right)$ for the hydrophobic surface $\left(\theta_{A}=107^{\circ}\right.$ and $\left.\theta_{R}=103^{\circ}\right)$. $(\mathbf{B})$ The diameter of droplets at the onset of droplet departure $\left(D_{o}\right)$ is depicted for three different surfaces at different jet Reynolds numbers $\left(R e_{j}\right)$. (C) For a comparison purpose, ratio of droplet diameter at a reference location $\left(s_{r}=1 \mathrm{~mm}\right)$ from the center of the impingement region to that at the onset of motion $\left(D / D_{o}\right)$ is plotted against the jet Reynolds numbers $\left(R e_{j}\right)$ for three different surfaces. (D) a reference apparent speed of droplets $\left(v_{r}=s_{r} /\left(t_{r}-t_{o}\right)\right)$ at a reference location $\left(s_{r}=1 \mathrm{~mm}\right)$ is plotted against the jet Reynolds numbers $\left(R e_{j}\right)$ for three different surfaces. The jet mean velocities were between $15 \mathrm{~m} / \mathrm{s}$ and $75 \mathrm{~m} / \mathrm{s}$.

motion, $D_{0}$, is used in place of the jet diameter, $\left(t-t_{0}\right) V_{j} / D_{0}$. The dynamics of drop motion are clearly quite complicated in this process.

Figure 7B, the diameter of droplets is presented at the onset of departure over a range of jet Reynolds numbers for three different surfaces: hydrophobic, hydrophilic and microstructured hydrophilic. For the same jet Reynolds number, the hydrophobic surface, which has the largest advancing contact line and smallest contact angle hysteresis, consistently shows the smallest critical droplet diameter for the onset of droplet motion. With decreasing advancing contact angle and contact angle hysteresis, the critical droplet diameter increases for a given jet Reynolds number with the microstructured hydrophilic surface consistently showing the largest values of critical diameter needed to induce droplet motion. Importantly, the critical droplet diameter can also be tuned by controlling the jet parameters, namely the jet velocity or Reynolds number. As can be seen in Fig. 7B, increasing jet Reynolds number results in a significant decrease in critical droplet diameter. At large values of jet Reynolds number, a distinct scaling can be observed that suggests, $D_{0} \sim R e_{j}^{-2}$. To investigate this further, forces acting on the droplet at force equilibrium are analyzed just before droplet departure.

The dominating forces are surface tension force along the droplet's contact line and aerodynamic drag forces on the droplet surface. Viscous friction is assumed to be negligible because the droplet is static at this period. The surface tension force along the contact line can be written as ${ }^{79}$

$$
F_{s}=\Gamma_{L} D_{o} \gamma\left(\cos \theta_{R}-\cos \theta_{A}\right)
$$

where $\Gamma_{L}$ is a contact line shape factor and $\gamma$ is the interfacial surface tension. The value of the contact line shape factor depends on the droplet geometry and symmetry. In general, its value has been experimentally found to lie within $1 / 2$ and $\pi / 2^{80,81}$. It has been observed that the state of motion of a droplet affects the surface tension 
force acting on a droplet, e.g. static and dynamic behavior ${ }^{82}$. Additionally, external vertical forces on the droplet can play a role in pinning the droplet ${ }^{83}$. Rather than resorting to a different mathematical description as suggested by Tadmor et al. ${ }^{84}$, Eq. (7) is used and the mentioned effects are absorbed in the order one contact line shape factor, $\Gamma_{L}$.

At equilibrium, the contact line force is balanced by the aerodynamics force generated by impinging jet that is trying to dislodge the droplet. The effects of velocity variation across the boundary layer can be ignored as the droplets are significantly larger than the boundary layer thickness (see supplementary material section $\mathrm{S}-5)^{29,79,85,86}$. Thus, the drag force, which is proportional to the stagnation pressure on the droplet, can be written as

$$
F_{d}=\frac{1}{2} \rho_{g} \Gamma_{A} C_{d} u_{o}^{2} D_{o}^{2}
$$

where $C_{d}$ is the drag coefficient of the droplet, $\rho_{g}$ is the surrounding gas density, $\Gamma_{A}$ is a shape factor of the projected area of the droplet in the flow direction (see supplementary material section S-5), and $u_{o}$ is the effective air velocity around the droplet. Note that $u_{o}$ scales with the jet mean velocity, $u_{o} \sim v_{j}$, and decays with distance from the location of jet impingement. Balancing the forces acting on a droplet at the onset of motion, yields the following relation.

$$
D_{o}=\frac{\gamma\left(\cos \theta_{R}-\cos \theta_{A}\right) \Gamma_{L}}{\frac{1}{2} \rho_{g} C_{d} \Gamma_{A}} \frac{1}{v_{j}^{2}} \sim \frac{1}{R e_{j}^{2}}
$$

Note that the scaling of critical diameter with jet Reynolds number predicted by Eq. (9) fits the experimental data in Fig. 7B quite well for the high jet Reynolds number cases of all three surfaces tested. However, a deviation is observed at the lowest jet Reynolds number studied. This is likely attributable to the complexity of evaluating the geometrical shape factors and the drag coefficient which are not constant as assumed her, but depend on the air velocity and diameter of the drop ${ }^{31}$.

Following the onset of droplet motion, the droplets grow through a combination of continued condensation, which is slow, and coalescence with smaller stationary droplets in their path, which is fast. At least initially, increasing droplet size is observed to result in an acceleration of the drop and an increased droplet velocity. As shown by Eq. (7), the interfacial retention force increases with $F_{s} \sim D$, while the aerodynamic drag force in Eq. (8) scales increases with $F_{d} \sim D^{2}$. Hence, as the droplets coalesce and grow beyond the critical diameter for droplet motion where these two forces are in balance, a force imbalance favoring aerodynamic drag over retention force exists and the droplets accelerate. In order to quantify the rate of droplet diameter growth during this acceleration period, the droplet diameter normalized by the critical diameter, $D_{r} / D_{o}$, was measured at a reference location $\left(s_{r}=1 \mathrm{~mm}\right)$ and is plotted against the jet Reynolds number for each of the three surfaces. A schematic diagram is shown in in Fig. 7D as a reference. The reference location lies within the accelerating period (Period II) for all the cases presented. In Fig. 7C, the diameter of each droplets was found to increases significantly by the time it reached a position $1 \mathrm{~mm}$ from the center of the impinging jet. The hydrophilic structured surface, which had the largest contact angle hysteresis shows the smallest increase in droplet diameter with an average of just $D_{r} / D_{o}=1.35$. This diameter increase suggests a volume increase of roughly $2.5 \mathrm{x}$ meaning that on average 2.5 coalescence events with similar sized drops have occured during the first $1 \mathrm{~mm}$ of travel. Within uncertainty, no significant difference between the hydrophilic and the hydrophobic cases could be observed. On average, the droplets on these surface grew faster than the microstructured hydrophilic surface with an increase of in droplet diameter of roughly $D_{r} / D_{o}=1.7$ and a corresponding volume change of $5 \mathrm{x}$. It is interesting to note that even though the size of droplets is different under varying jet Reynolds numbers, the ratio of increase is constant for the same surface wettability independent of jet Reynolds number. This suggests a self-similarity property of the condensation process.

In Fig. 7D, the velocity of the droplets, $v_{r}$ is presented as a function of jet Reynolds number. Here, again the velocity is measured at a location within the accelerating region $\left(s_{r}=1 \mathrm{~mm}\right)$. The droplet velocity can be seem to vary monotonically with the jet Reynolds number. Moreover, the droplet apparent speeds are similar for the three surfaces within the experimental uncertainty. A simplistic equation of motion (EOM) is given in supplementary material section S.6. In the acceleration period, the drag force is assumed to be much greater than the retention forces. This in turn means that the droplet motion in Period II should be independent of the surface wettability which agrees with our experimental observation in Fig. 7D.

\section{Discussion}

A novel continuous drop-wise condensation that is efficient and compact was achieved by utilizing jet impingement of water vapor on a cooled surface. The fluid dynamics of an impinged jet showed an excellent shedding capability to overcome the limitations of the state-of-the-art techniques.

In summary, we have demonstrated the capability of our CDC design in improving the condensation process substantially compared to state-of-the-art condensers and humidification technologies. We performed condensation experiments on modified and unmodified Silicon substrates on a broad range of contact angles. The drop size being shed was controlled by tuning the jet parameters namely the jet velocity in this work. We showed that micron-sized droplets could be shed effectively even on hydrophilic surfaces.

By comparing the condensation rate per unit volume of state-of-the-art dehumidification technologies, we showed that our design is at least six fold higher. This significant increase is attributed to the thinning of the diffusion layer which is known to impede vapor condensation. We also illustrated that by controlling the maximum 
droplet size being shed, improvements as high as $375 \%$ in heat flux was possible for steam condensers in the absence of NCG.

Finally, we discussed the droplet dynamics and growth under the jet impingement action. By comparing the different forces acting on a droplet, we were able to predict the size of droplet being shed under varying jet Reynolds number and surface wettability. Furthermore, the models presented in this work are the starting point for further optimization of the design to obtain more compact dehumidification using CDC technique.

Further discussion. The droplets generated within the impingement area are of sizes less than the capillary length of water (or bond number $B o<<0.1$ ). Therefore, gravitational force effect is negligible which in turn means the current analysis is independent of surface orientation. Gravitational force will be effective on the stationary droplets which reside in the region corresponding to the decelerating period (Period III, Fig. 6B). The effect of gravitational force as well as other dominating forces, such as viscous dissipation are subjects of future research.

It was noticed that in surfaces that are characterized by high contact angle hystereses droplets tend to stop at random locations corresponding to the decelerating period ( Period III in Fig. 6B). This indicates that the droplet shedding advantage of jet impingement is lost away from the impingement region. We believe that this does not undermine the effectiveness of CDC as most of vapor condensation takes place where shedding is significant. To overcome the accumulation of condensate in that region, different engineering solutions could be applied. Placing the condensation surface vertically helps with shedding larger droplets. It is experimentally observed that stationary droplets in regions corresponding to period III grow quickly by droplet feeding coming from the impingement region. Other methods, such as surface texturing or capillary wicking could be utilized to effectively drain excessive condensate.

Surfaces with low contact angle hystereses tend to show better uniformity of droplet shedding and stoppage location. The shedding of droplets is further improved by droplet jumping in the case of superhydrophobic surface $\left(\theta_{A}=157^{\circ}\right.$ and $\left.\theta_{R}=154^{\circ}\right)$. As noticed in Fig. 3E and video S.6, droplets in the micro-scale jump off the surface due to the release of energy upon coalescence. This phenomenon has been shown earlier to have potential in improving $\mathrm{DWC}^{25}$ and energy harvesting ${ }^{87}$. A great advantage offered by CDC is that jumping droplets are further shed by flow generated from jet impingement. Hence, mitigate the return of micro-droplets on the surface and therefore prevent flooding of the surface.

Supplementary Material are presented in the attached file.

\section{Materials and Methods}

Surface preparation and characterization. Commercially available Silicon wafers (Techgophers) were used as the base condensation surfaces. In this work, we utilized five surface modifications namely (1) Hydrophilic Si surface, (2) hydrophilic micro-structured Si surface, and (3) hydrophobic silanized Si surface (4) superhydrophobic micro-structured Si surface, and (5) superhydrophobic spray-coated Si surface. Silicon wafers of similar thicknesses were used in this work to provide similar thermal resistances over the different tests. Checking the temperature distribution on the Si surfaces shows that their thermal resistance is quite negligible compared to the vapor-NCG side dominating thermal resistance. Before each experiment, the surfaces were cleaned with acetone (J.T.Baker), isopropanol (J.T.Baker), ethanol (J.T.Baker) and DI water and dried with filtered nitrogen stream. Experiments were done immediately after the cleaning process so that the effect of organic compounds found in room environment is negligible.

After $\mathrm{Si}$ wafers were cleaned different methods were utilized to change their wettability. The untreated $\mathrm{Si}$ wafer provides the base surface which happens to be slightly hydrophilic $\left(\theta_{A}=85^{\circ}\right.$ and $\left.\theta_{R}=72^{\circ}\right)$. For altering the surface wettability, extra steps were performed other than the cleaning process. Micro-posts were fabricated on top of the silicon wafer with a diameter of $6 \mu \mathrm{m}$, height of $6 \mu \mathrm{m}$, and pitch of $50 \mu \mathrm{m}$. Because water condensation exists between the posts, a Wenzel state is noticed which renders the silicon wafer more hydrophilic $\left(\theta_{A}=\right.$ $70^{\circ}$ and $\left.\theta_{R}=50^{\circ}\right)$. To render the substrate hydrophobic $\left(\theta_{A}=107^{\circ}\right.$ and $\left.\theta_{R}=103^{\circ}\right)$, the base surface was silanized with a thin layer of polydimethylsiloxane (PDMS) using a conventional dip coating method. The film thickness is negligible compared to the thickness of the silicon wafer and therefore does not impede the heat transfer rate. Micro-posts were fabricated on top of the silicon wafer with a diameter of $40 \mu \mathrm{m}$, height $40 \mu \mathrm{m}$, and pitch of 50 $\mu \mathrm{m}$. To obtain superhydrophibicity, a combination of silanization process with the roughened surface yielded a superhydrophobic surface with a high contact angle hysteresis $\left(\theta_{A}=160^{\circ}\right.$ and $\left.\theta_{R}=127^{\circ}\right)$. For the last surface, we utilized an aerosol spray coating method to coat the silicon wafer with WX2100 (purchased from cytonix) in which Fluorothane is the active ingredient. The resultant surface is superhydrophobic with negligible contact angle hysteresis $\left(\theta_{A}=157^{\circ}\right.$ and $\left.\theta_{R}=154^{\circ}\right)$. The contact angle is independent of the coating thickness. Therefore, we coated the surface with about $50 \mu \mathrm{m}$ without significant impedance of heat transfer. SEM images are shown in Fig. S2 for detailed surface topography (supplementary material).

The surface wettability was characterized before and after each experimental run to assess the homogeneity of their wettability as well as the consistency of contact angle measurements. Droplet shape analyzer (KRÜ SS, DSA 100) was used for contact angle measurements. The static advancing and receding contact angles were measured using the protocol outlined in this paper ${ }^{88}$. Measurements were repeated on different spots of the $\mathrm{Si}$ wafers to ensure homogeneity and consistency. Droplets with diameters less than the capillary length were tested to ensure negligible effects of gravity. Static advancing and receding contact angles are summarized in Table 1.

Condensation experiments. Figure S1, we show a schematic of the experimental setup which consists of a bubble humidifier, a flow system, and a cooled surface. Dry filtered air was bubbled into a pool of roomtemperature DI water through several spargers (Ferroday). The spargers generate micron-sized bubbles which 
due to their high contact area with water get humidified to above $95 \%$. The humid air generated exists at a room temperature $\left(T_{\infty}=21^{\circ} \mathrm{C} \pm 1{ }^{\circ} \mathrm{C}\right)$ and ambient humidity of $(60 \% \pm 1 \%)$. Humidity of ambient air and humidified jet was measured using a Hygrometer (VWR). The humidified air was led through a tube (Mc-MASTER-CARR) of inner diameter $(D=0.047$ in) to impinge normally on the cooled surface. For experimental convenience, the tube was bend to $90^{\circ}$ while allowing enough length $(L=0.84$ in) before the exist section ensuring fully developed flow beyond the secondary flow region. The flow rate of humidified air was controlled by flow-adjustment valve and measured using a rotameter (OMEGA, model no. FMA-A2323). Volumetric flow rates tested range from 1 LPM to 5 LPM. The corresponding jet mean velocities range from $15 \mathrm{~m} / \mathrm{s}$ to $75 \mathrm{~m} / \mathrm{s}$. The humid air jet exits the tube at a standoff distance ( $H=0.32$ in).

The condensation surface was the different Silicon wafers described earlier. The surfaces were placed on an Aluminum substrate with a thermally conductive paste in between. The Aluminum substrate was placed on the cold side of a Peltier plate with a thermally conductive paste. A simple peltier plate with a temperature controller unit was used to maintain a constant surface temperature $\left(T_{s}=15^{\circ} \mathrm{C} \pm 1{ }^{\circ} \mathrm{C}\right)$. An Infra-red (IR) camera (FLIR, A6753sc), and two flush-mounted k-type thermocouples (OMEGA, HH378) were used to observe the condensation substrate temperature as well as the condensate droplets. The substrate temperatures measured by the three sensors were in agreement within $1{ }^{\circ} \mathrm{C}$. This ruled out any possible temperature variation on the surface and ensured that the thermal resistance of vapor-gas side was dominant.

Systematic experiments were performed by first adjusting the flow to the desired jet Reynolds number $R e_{j}=4 Q / \pi \nu D$, where $v$ is the kinematic viscosity of humid air. Then, the surface temperature was set to the desired temperature. The condensation process was allowed to reach a quasi-steady state by waiting for about 15 minutes before taking experimental measurements. To visualize the condensation process an optical microscope (Nikon, AZ100) with a high-speed camera (Photron, FASTCAM Nova) were used.

\section{Data availability}

Additional data and materials are available online.

Received: 26 March 2021; Accepted: 13 September 2021

Published online: 04 October 2021

\section{References}

1. Jadhav, S., Morey, P., Karpe, M. \& Kadam, V. Dip pen nanolithography. Am. J. PharmTech Res. 2, 148-168 (2012).

2. Enright, R., Miljkovic, N., Alvarado, J. L., Kim, K. \& Rose, J. W. Dropwise condensation on micro-and nanostructured surfaces. Nanosc. Microsc. Thermophys. Eng. 18, 223-250 (2014).

3. Wilson, J. G. The principles of cloud-chamber technique (Cambridge University Press, 2014).

4. Zhang, A., Bai, H. \& Li, L. Breath figure: a nature-inspired preparation method for ordered porous films. Chem. Rev. 115, 9801-9868 (2015).

5. Warsinger, D. M., Mistry, K. H., Nayar, K. G., Chung, H. W. \& Lienhard, J. H. Entropy generation of desalination powered by variable temperature waste heat. Entropy 17, 7530-7566 (2015).

6. Miljkovic, N., Preston, D. J. \& Wang, E. N. Recent developments in altered wettability for enhancing condensation. In Encyclopedia of Two-Phase Heat Transfer and Flow II: Special Topics and Applications 85-131 (World Scientific, 2016).

7. Chen, Y., Yang, S., Wu, J. \& Zhou, J. Impact of helical baffle structure on heat transfer performance of vertical condensers. Appl. Therm. Eng. 115, 9-16 (2017).

8. Li, S. et al. The influence of structural parameters on heat transfer and pressure drop for hydrocarbon mixture refrigerant during condensation in enhanced spiral pipes. Appl. Therm. Eng. (2018).

9. Zhao, Y. et al. Effects of millimetric geometric features on dropwise condensation under different vapor conditions. Int. J. Heat Mass Transf. 119, 931-938 (2018).

10. Famileh, I. Z. \& Esfahani, J. A. Experimental investigation of wet flue gas condensation using twisted tape insert. Int. J. Heat Mass Transf. 108, 1466-1480 (2017).

11. Datt, R., Bhist, M. S., Kotiyal, A. D., Maithani, R. \& Kumar, A. Development of new correlations for heat transfer and friction loss of solid ring with combined square wing twisted tape inserts heat exchanger tube. Experiment. Heat Transf. .

12. Rashidi, S., Mahian, O. \& Languri, E. M. Applications of nanofluids in condensing and evaporating systems. J. Therm. Anal. Calorim. 131, 2027-2039 (2018)

13. Shahriari, A., Birbarah, P., Oh, J., Miljkovic, N. \& Bahadur, V. Electric Field-Based Control and Enhancement of Boiling and Condensationhttps://doi.org/10.1080/15567265.2016.1253630 (2017).

14. Miljkovic, N., Preston, D. J., Enright, R. \& Wang, E. N. Electric-field-enhanced condensation on superhydrophobic nanostructured surfaces. ACS Nano7, 11043-11054. https://doi.org/10.1021/nn404707j (2013). 0706.1062v1.

15. Sett, S. et al. Transient pulse condensation. Appl. Phys. Lett. 117, 091602 (2020).

16. Daniel, D., Mankin, M. N., Belisle, R. A., Wong, T.-S. \& Aizenberg, J. Lubricant-infused micro/nano-structured surfaces with tunable dynamic omniphobicity at high temperatures. Appl. Phys. Lett. 102, 231603 (2013).

17. Cho, H. J., Preston, D. J., Zhu, Y. \& Wang, E. N. Nanoengineered materials for liquid-vapour phase-change heat transfer. Nat. Rev. Mater. 2, 16092 (2017).

18. Ahlers, M., Buck-Emden, A. \& Bart, H.-J. Is dropwise condensation feasible? a review on surface modifications for continuous dropwise condensation and a profitability analysis. J. Adv. Res. 16, 1-13 (2019).

19. Ma, J., Sett, S., Cha, H., Yan, X. \& Miljkovic, N. Recent developments, challenges, and pathways to stable dropwise condensation: A perspective. Appl. Phys. Lett. 116, 260501 (2020).

20. Rose, J. Dropwise condensation theory and experiment: A review. Proc. Inst. Mech. Eng. Part A: J. Power Energy 216, 115-128 (2002).

21. Wen, R., Ma, X., Lee, Y.-C. \& Yang, R. Liquid-vapor phase-change heat transfer on functionalized nanowired surfaces and beyond. Joule 2, 2307-2347 (2018).

22. Rose, J. W. Personal reflections on fifty years of condensation heat transfer research. Journal of Enhanced Heat Transfer 22, (2015).

23. Kim, S. \& Kim, K. J. Dropwise condensation modeling suitable for superhydrophobic surfaces. Journal of heat transfer 133, (2011).

24. Dimitrakopoulos, P. \& Higdon, J. On the gravitational displacement of three-dimensional fluid droplets from inclined solid surfaces. J. Fluid Mech. 395, 181-209 (1999).

25. Miljkovic, N. et al. Jumping-droplet-enhanced condensation on scalable superhydrophobic nanostructured surfaces. Nano Lett. 13, 179-187 (2012). 
26. Yuan, Z., Hu, Z., Chu, F. \& Wu, X. Enhanced and guided self-propelled jumping on the superhydrophobic surfaces with macrotexture. Appl. Phys. Lett. 115, 163701 (2019).

27. Peng, Q. et al. Breaking droplet jumping energy conversion limits with superhydrophobic microgrooves. Langmuir 36, 9510-9522 (2020).

28. Hu, H., Huang, S. \& Chen, L. Displacement of liquid droplets on micro-grooved surfaces with air flow. Exp. Thermal Fluid Sci. 49, 86-93 (2013).

29. Roisman, I. V., Criscione, A., Tropea, C., Mandal, D. K. \& Amirfazli, A. Dislodging a sessile drop by a high-reynolds-number shear flow at subfreezing temperatures. Phys. Rev. E 92, 023007 (2015).

30. Seevaratnam, G., Ding, H., Michel, O., Heng, J. \& Matar, O. Laminar flow deformation of a droplet adhering to a wall in a channel. Chem. Eng. Sci. 65, 4523-4534 (2010).

31. Milne, A. \& Amirfazli, A. Drop shedding by shear flow for hydrophilic to superhydrophobic surfaces. Langmuir 25, 14155-14164 (2009).

32. Razzaghi, A., Banitabaei, S. \& Amirfazli, A. Shedding of multiple sessile droplets by an airflow. Phys. Fluids 30, 087104 (2018).

33. Yan, X. et al. Near field condensation. (2020).

34. Li, J. \& Guo, Z. Spontaneous directional transportations of water droplets on surfaces driven by gradient structures. Nanoscale 10, 13814-13831 (2018).

35. Graham, C. \& Griffith, P. Drop size distributions and heat transfer in dropwise condensation. Int. J. Heat Mass Transf. 16, 337-346 (1973).

36. Wen, R. et al. Wetting transition of condensed droplets on nanostructured superhydrophobic surfaces: coordination of surface properties and condensing conditions. ACS applied materials \& interfaces $9,13770-13777$ (2017).

37. Citakoglu, E. \& Rose, J. Dropwise condensation-some factors influencing the validity of heat-transfer measurements. Int. J. Heat Mass Transf. 11, 523-537 (1968).

38. Alshehri, A., Andalib, S. \& Kavehpour, H. P. Numerical modeling of vapor condensation over a wide range of non-condensable gas concentrations. Int. J. Heat Mass Transf. 151, 119405 (2020).

39. Zhang, J. X. \& Wang, L. Effect of air on condensation in a non-vacuum gravity heat pipe. Appl. Therm. Eng. 114, 255-263. https:// doi.org/10.1016/j.applthermaleng.2016.11.209 (2017).

40. Narayan, G. P. et al. The potential of solar-driven humidification-dehumidification desalination for small-scale decentralized water production. Renew. Sustain. Energy Rev. 14, 1187-1201 (2010).

41. Giwa, A., Akther, N., Al Housani, A., Haris, S. \& Hasan, S. W. Recent advances in humidification dehumidification (hdh) desalination processes: Improved designs and productivity. Renew. Sustain. Energy Rev. 57, 929-944 (2016).

42. Srithar, K. \& Rajaseenivasan, T. Recent fresh water augmentation techniques in solar still and hdh desalination-a review. Renew. Sustain. Energy Rev. 82, 629-644 (2018).

43. Chafik, E. Design of plants for solar desalination using the multi-stag heating/humidifying technique. Desalination 168, 55-71 (2004).

44. Chafik, E. A new type of seawater desalination plants using solar energy. Desalination 156, 333-348 (2003).

45. Farid, M., Parekh, S., Selman, J. \& Al-Hallaj, S. Solar desalination with a humidification-dehumidification cycle: mathematical modeling of the unit. Desalination 151, 153-164 (2003).

46. Chang, Z., Zheng, H., Yang, Y., Su, Y. \& Duan, Z. Experimental investigation of a novel multi-effect solar desalination system based on humidification-dehumidification process. Renewable Energy 69, 253-259 (2014).

47. Klausner, J. F., Li, Y. \& Mei, R. Evaporative heat and mass transfer for the diffusion driven desalination process. Heat Mass Transf. 42, $528(2006)$

48. Dawoud, B., Zurigat, Y., Klitzing, B., Aldoss, T. \& Theodoridis, G. On the possible techniques to cool the condenser of seawater greenhouses. Desalination 195, 119-140 (2006).

49. Hu, T., Hassabou, A. H., Spinnler, M. \& Polifke, W. Performance analysis and optimization of direct contact condensation in a pcm fixed bed regenerator. Desalination 280, 232-243 (2011).

50. Agboola, O. P. \& Egelioglu, F. An empirical evaluation of an integrated inclined solar water desalination system with spray jets variation. Desalin. Water Treat. 53, 2875-2881 (2015)

51. Tow, E. W. et al. Experiments and modeling of bubble column dehumidifier performance. Int. J. Therm. Sci. 80, 65-75 (2014).

52. Liu, H. \& Sharqawy, M. H. Experimental performance of bubble column humidifier and dehumidifier under varying pressure. Int. J. Heat Mass Transf. 93, 934-944 (2016).

53. Sadeghpour, A. et al. Water vapor capturing using an array of traveling liquid beads for desalination and water treatment. Sci. Adv. 5, eaav7662 (2019).

54. Narayan, G. P., McGovern, R. H., Lienhard, J. H. \& Zubair, S. M. Helium as a carrier gas in humidification dehumidification desalination systems. (2011).

55. Arabi, M. K. A. \& Reddy, K. V. Performance evaluation of desalination processes based on the humidification/dehumidification cycle with different carrier gases. Desalination 156, 281-293 (2003).

56. Raben, IA and Commerford, George and Dietert, R. An investigation of the use of acoustic vibrations to improve heat transfer rates and reduce scaling in distillation units used for saline water conversion. Available from the National Technical Information Service, Springfield VA 22161 as PB-171 911, Price codes: A 08 in paper copy, A 01 in microfiche. OSW Research and Development Progress Report49 (1961).

57. Chen, X., Doughramaji, N., Betz, A. R. \& Derby, M. M. Droplet ejection and sliding on a flapping film. AIP Adv. 7, 035014 (2017).

58. Migliaccio, C. P. Resonance-induced condensate shedding for high-efficiency heat transfer. Int. J. Heat Mass Transf. 79, 720-726 (2014).

59. Moradi, M., Chini, S. F. \& Rahimian, M. H. Vibration-enhanced condensation heat transfer on superhydrophobic surfaces: An experimental study. AIP Adv. 10, 095123 (2020).

60. Velkoff, H. \& Miller, J. Condensation of vapor on a vertical plate with a transverse electrostatic field. J. Heat Transf. 87, 197-201 (1965).

61. Baratian, D., Dey, R., Hoek, H., Van Den Ende, D. \& Mugele, F. Breath figures under electrowetting: electrically controlled evolution of drop condensation patterns. Tech. Rep. (2018). 1712.03059v5.

62. Dey, R. et al. Controlling shedding characteristics of condensate drops using electrowetting. Appl. Phys. Lett. 113, 243703. https:// doi.org/10.1063/1.5064363 (2018).

63. Traipattanakul, B., Tso, C. \& Chao, C. Y. Electrostatic-induced coalescing-jumping droplets on nanostructured superhydrophobic surfaces. Int. J. Heat Mass Transf. 128, 550-561. https://doi.org/10.1016/j.ijheatmasstransfer.2018.08.134 (2019).

64. Mohamed, H. A. Effect of rotation and surface roughness on heat transfer rate to flow through vertical cylinders in steam condensation process. (2006).

65. Peng, S. Theoretical analysis of laminar film condensation in a rotating cylinder with a scraper. Heat Mass Transf. 34, 279-285 (1998).

66. Yanniotis, S. \& Kolokotsa, D. Experimental study of water vapour condensation on a rotating disc. Int. Commun. Heat Mass Transfer 23, 721-729 (1996).

67. Viskanta, R. Heat transfer to impinging isothermal gas and flame jets. Exp. Thermal Fluid Sci. 6, 111-134 (1993). 
68. Lienhard, J. H. Heat transfer by impingement of circular free-surface liquid jets. In 18th National and 7th ISHMT-ASME, Heat and Mass Transfer Conference, Guwahati, India (2006).

69. Mujumdar, A. S. 15 impingement drying. Handbook of Industrial Drying 371 (2014).

70. Qiu, L., Dubey, S., Choo, F. H. \& Duan, F. Recent developments of jet impingement nucleate boiling. Int. J. Heat Mass Transf. 89, 42-58 (2015).

71. Khangembam, C., Singh, D., Handique, J. \& Singh, K. Experimental and numerical study of air-water mist jet impingement cooling on a cylinder. Int. J. Heat Mass Transf. 150, 119368 (2020).

72. Ji, D.-Y., Kim, D. \& Lee, K.-Y. Enhancement of condensation heat transfer in the presence of non-condensable gas using steam jet method. Int. J. Heat Mass Transf. 130, 603-612 (2019).

73. Ji, D.-Y., Lee, J.-W., Kim, D., Hwang, W. \& Lee, K.-Y. Effective reduction of non-condensable gas effects on condensation heat transfer: Surface modification and steam jet injection. Appl. Therm. Eng.115264, (2020).

74. Alshehri, A. \& Kavehpour, H. P. Breath figure spot: a recovery concentration manifestation. Int. J. Heat Mass Transf. 172, 121166. https://doi.org/10.1016/j.ijheatmasstransfer.2021.121166 (2021).

75. Jambunathan, K., Lai, E., Moss, M. \& Button, B. A review of heat transfer data for single circular jet impingement. Int. J. Heat Fluid Flow 13, 106-115 (1992).

76. Hollworth, B. \& Wilson, S. Entrainment effects on impingement heat transfer. i measurements of heated jet velocity and temperature distributions, and recovery temperatures on target surface. In ASME and American Institute of Chemical Engineers, Heat Transfer Conference (1983).

77. Sievers, M. \& Lienhard, J. H. Design of flat-plate dehumidifiers for humidification-dehumidification desalination systems. Heat Transf. Eng. 34, 543-561 (2013).

78. Le Fevre, E. \& Rose, J. W. A theory of heat transfer by dropwise condensation. In International Heat Transfer Conference Digital Library (Begel House Inc., 1966).

79. Furmidge, C. Studies at phase interfaces. i. the sliding of liquid drops on solid surfaces and a theory for spray retention. J. Colloid Sci. 17, 309-324 (1962).

80. Extrand, C. \& Gent, A. Retention of liquid drops by solid surfaces. J. Colloid Interface Sci. 138, 431-442 (1990).

81. ElSherbini, A. \& Jacobi, A. Retention forces and contact angles for critical liquid drops on non-horizontal surfaces. J. Colloid Interface Sci. 299, 841-849 (2006).

82. Gao, N. et al. How drops start sliding over solid surfaces. Nat. Phys. 14, 191-196 (2018).

83. Tadmor, R. et al. Measurement of lateral adhesion forces at the interface between a liquid drop and a substrate. Phys. Rev. Lett. 103, 266101 (2009).

84. Tadmor, R. Approaches in wetting phenomena. Soft Matter 7, 1577-1580 (2011).

85. Dussan, E. et al. On the ability of drops or bubbles to stick to non-horizontal surfaces of solids. part 2. small drops or bubbles having contact angles of arbitrary size. J. Fluid Mech. 151, 20 (1985).

86. Dussan, V. et al. On the ability of drops to stick to surfaces of solids. iii: The influences of the motion of the surrounding fluid on dislodging drops. J. Fluid Mech. 174, 381-397 (1987).

87. Miljkovic, N., Preston, D. J., Enright, R. \& Wang, E. N. Jumping-droplet electrostatic energy harvesting. Appl. Phys. Lett. 105, 013111. https://doi.org/10.1063/1.4886798 (2014).

88. Huhtamäki, T., Tian, X., Korhonen, J. T. \& Ras, R. H. Surface-wetting characterization using contact-angle measurements. Nat. Protoc. 13, 1521-1538 (2018).

\section{Acknowledgements}

A. Alshehri would like to express his sincere gratitude to King Fahd University of Petroleum and Minerals (KFUPM), Dhahran, Saudi Arabia.

\section{Author contributions}

A.A.: Conceptualization, Methodology, Formal analysis, Experiments, Investigation, Visualization, Writing. J.P.R.: Formal analysis, Discussions, Investigation, Writing. H.P.K.: Discussions, Investigation, Supervision, Funding acquisition, Editing. All authors reviewed the manuscript.

\section{Funding}

This research was supported by the National Science Foundation under Grant No. CBET-2032533.

\section{Competing Interests}

The authors declare no competing interests.

\section{Additional information}

Supplementary Information The online version contains supplementary material available at https://doi.org/ 10.1038/s41598-021-98992-9.

Correspondence and requests for materials should be addressed to A.A.

Reprints and permissions information is available at www.nature.com/reprints.

Publisher's note Springer Nature remains neutral with regard to jurisdictional claims in published maps and institutional affiliations.

Open Access This article is licensed under a Creative Commons Attribution 4.0 International License, which permits use, sharing, adaptation, distribution and reproduction in any medium or format, as long as you give appropriate credit to the original author(s) and the source, provide a link to the Creative Commons licence, and indicate if changes were made. The images or other third party material in this article are included in the article's Creative Commons licence, unless indicated otherwise in a credit line to the material. If material is not included in the article's Creative Commons licence and your intended use is not permitted by statutory regulation or exceeds the permitted use, you will need to obtain permission directly from the copyright holder. To view a copy of this licence, visit http://creativecommons.org/licenses/by/4.0/.

(C) The Author(s) 2021 